\title{
Research Of Power System Load Model Based on Improved Genetic Programming
}

\author{
Jun Wang ${ }^{1, a}$, Jian Zhang ${ }^{1, b}$ \\ ${ }^{1}$ School of Electrical Engineering, Zhengzhou University, Zhengzhou 450000,China; \\ a394893525@qq.com, bzhangjian63@zzu.edu.cn
}

\begin{abstract}
Keywords:power system,load model,genetic programming,automatic modeling.
Abstract.The model of the power system load has an important impact on power flow calculation,stability analysis.Compared with the traditional load modeling method to determine the structure further identification of the model parameters, using the genetic programming to load modeling do not have to preset the model structure.It automatically generates a different function from the input and output variables.According to their fitness looking for the most accurate fit function.In this paper,using the improved method of GP to load modeling.Precision and efficiency has been improved by optimize the adaptation of the calculation process. The effectiveness and feasibility of the improved genetic programming method is verified by compare with the traditional load modeling method.
\end{abstract}

\section{Introduction}

Power system digital simulation plays an irreplaceable role in the operation and control of the power grid.Power system load modeling is the basis of the simulation, which aims to provide the model can reflect power system load characteristics. The results show that,in the process of load modeling, in order to effectively reflect the power system operating state,we should ensure the accuracy of the load model.So the model can applied to the research on power flow calculation, voltage stability analysis and reactive power compensation ${ }^{[1]}$.

The methods of load modeling mainly includes component-based modeling approach and measurement-based modeling approach.The component-based modeling approach have the advantages of simple and less knowledge dependency.But the measured data and model structure should be known when we identify the parameter ${ }^{[2]}$.In this paper,we use the improved Genetic Programming to load modeling.The Genetic Programming is the branch of evolutionary computation. We only need to know the noisy data when we identify the parameter.It's different form traditional modeling methods which also need to know the model structure.But Genetic Programming also have disadvantages such as the good model structure was eliminated because of the parameter stochastic property.

Therefore,we improve the Genetic Programming and compare with the traditional modeling methods.The effectiveness and feasibility of the improved GP is verified.It can provide a new approach to load modeling.

\section{Power system load model and improved genetic programming}

The load model is equations for describe load characteristic and it can be divided into static and dynamic according to the dynamic characteristic.It also can divided into mechanism model and non-mechanism model.Generally,we use algebraic equations to describe the static load model and differential equation to describe the dynamic load model.

Load model. The static load model can reflect the load power change with voltage and frequency.It can use in the process of power flow calculation and voltage stability analysis.The common load model has constant impedance model,polynomial model and power function model.The constant impedance model is the most basic load model.It has simple structure but has larger difference with real load characteristics. 
The forms of the load model are varied.There are constant impedance model,constant current model,power index model,polynomial model,induction motor load model and voltage-dependent load model.

In the traditional load modeling process, The model structure must be identify.In this paper,we established the load model library to find the optimal model.The load model library include power function load model,ZIP load model,Z+I load model,Z+P load model and P+I load model .

Improved genetic programming. The Genetic Programming reference from natural selection and genetic mechanism.It's based on the basic idea of Genetic Algorithm but using the hierarchical tree-structure ${ }^{[3]}$.Every tree-structure corresponds to a computer program in solution space.

The Genetic Programming will random generate the initial population according to the given issues.Every individual in population has the fitness which can evaluate its performance according to the principle of survival of the fittest, The GP generate the next population by genetic operation like cross, variation and replication.

In the Genetic Programming,we should know how to use tree-structure to represent program,the node of the tree is divided into two parts:the initial function and end point ${ }^{[5]}$. The function could be arithmetic operator $(+,-,,, / \ldots)$, the mathematical function( $\sin , \cos , \exp , \tan \ldots)$ and the Boolean operator(AND,OR,NOT ... ).Endpoint set $\mathrm{T}$ include all constants and variable like $\mathrm{T}=\{\mathrm{p}, \mathrm{x}\}$. The conditions for the termination judgment:the individual with the maximum fitness is optimal solution.

The GP modeling have the advantage of do not need determine the model structure ${ }^{[8]}$.But it also have disadvantage such as the good model structure may be eliminated because the randomness of parameter.So in this paper,the fitness of population in Genetic Programming is improved.we improved the population fitness. The constant term will be assignment in designed times rather than once, then choosing the maximum fitness individual as optimal solution.

\section{Load model based on improved genetic programming}

In order to verify the effectiveness of the improved GP.The original load data in calculation process all come from induction motor static voltage testing in the dynamic modeling laboratory ${ }^{[4]}$. The Genetic Programming get the optimal solution by automatic programming.First,Using standard GP to modeling in Matlab according to the data,the original model is as follows:

$$
\left\{\begin{array}{l}
P=(U s) * *((0.42745) . /((0.054501)+(U s))) \\
Q=((U s) . *((U s)-(0.76833)))+(0.34157)
\end{array}\right.
$$

The fitting error of the optimal load model is 0.043.To see the fitting degree more clear,The response curve of fitting and actual have been assigned as shown in Fig 1. We can see the load model of GP can fitting the actual model.

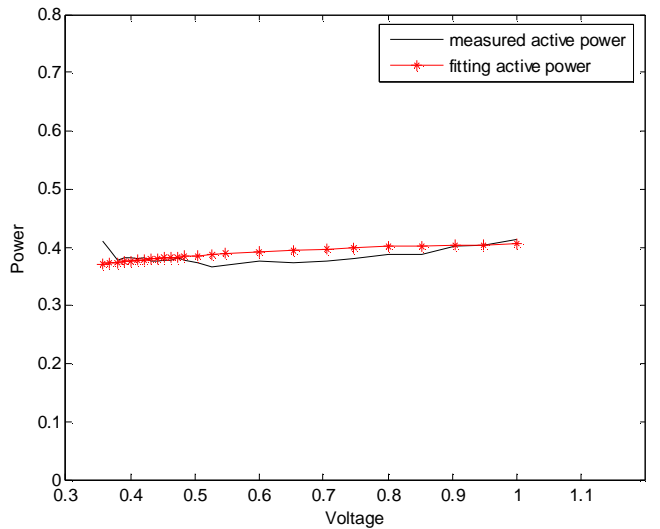

(a) active power

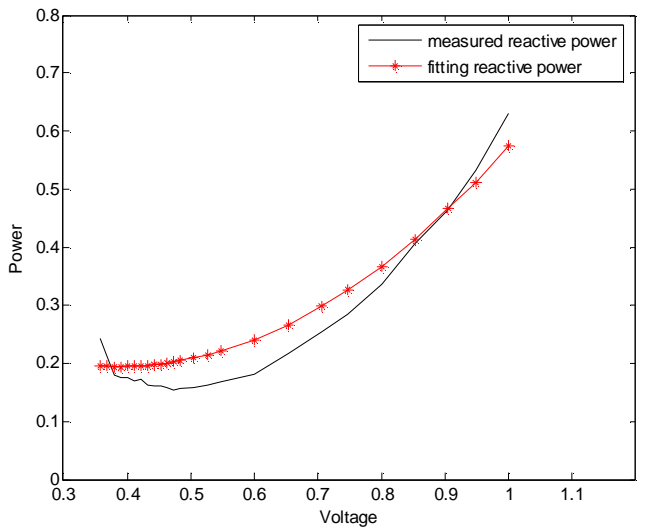

(b) reactive power

Fig.1 Voltage characteristics for standard GP 
In order to get the load model which can reflect the real load and apply to the power system simulation, we use improved GP to load modeling. The important part is detailed description in the last section.Now we run the load modeling program directly in the Matlab,the power system load model showed that:

$$
\left\{\begin{array}{l}
P=0.28655 U s^{2}-0.34984 U s+0.47880 \\
Q=1.84959 U s^{2}-1.83791 U s+0.61936
\end{array}\right.
$$

The fitting error of the optimal load model is 0.004 which is 1 to 2 order of magnitude lower than the former.The calculation results is ideal.To see the fitting degree more clear,The response curve of fitting and actual have been assigned as shown in Fig 2.We can see that the load model of GP can accurate curve-fitting the actual model.Therefore,the improved GP model have more accurate fitting degree than the traditional GP model.Because the model parameter assignment multiple times instead of once in the improved GP calculation process, it will reduce the modeling time and the neglected probability of the good model.The feasibility and effectiveness of improved GP is verified.

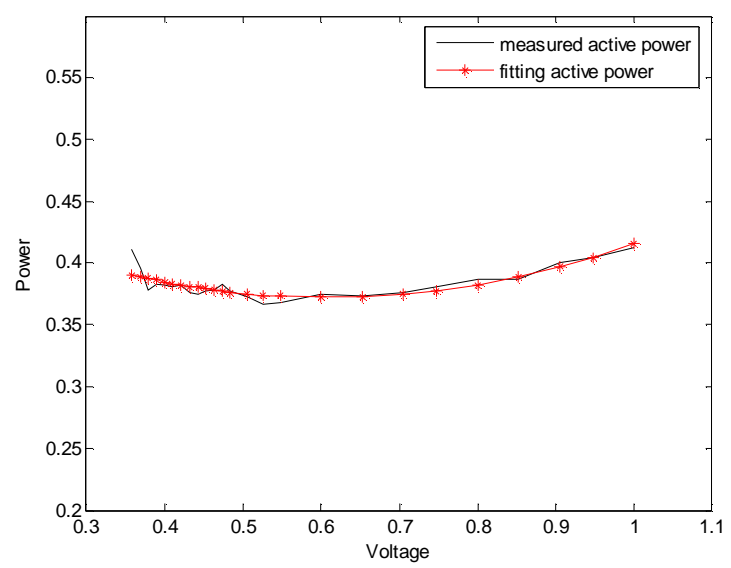

(a) active power

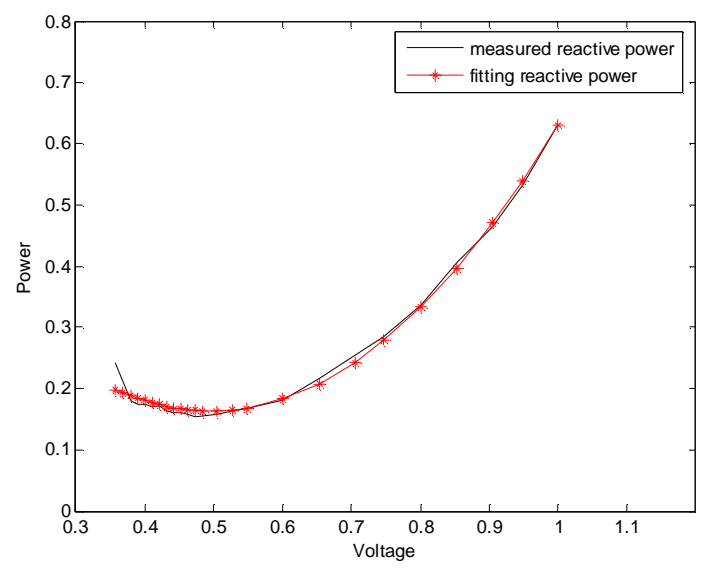

(b) reactive power

Fig.2 Voltage characteristics for improved GP

In the traditional way of load modeling,the model structure must be determine at first time,then identify the parameter.the composition of the load,the measured data and the load characteristic of power equipment must to know, when we determine the model structure. This method not only need to consider a lot of model combination ,but also need the professional skills.It's not easy to build ideal load model.However, the method which using computer search optimum model structure is more convenient than the traditional method and have more engineering significance and value.But the structure and fitting degree of the model should be further illustrates.

Now, The essay is to contrast respective characteristics between improved GP model and the traditional model from the model library which given in the first section of this paper.Because space is limited,We do not discuss the process of modeling and fitting curve here. The comparative analysis of model structure and error between traditional and GP methods are list out at Table 1.

Table1 The structure and error of load models

\begin{tabular}{cccccc}
\hline Model & Improved GP & power function & $\mathrm{Z}+\mathrm{I}$ & $\mathrm{Z}+\mathrm{P}$ & $\mathrm{P}+\mathrm{I}$ \\
\hline $\mathrm{P}$ & $\mathrm{P}=0.28655 \mathrm{U}^{2}-$ & $\mathrm{P}=0.39062 \mathrm{U}^{0.03118}$ & $\mathrm{P}=-0.88547 \mathrm{U}^{2}$ & $\mathrm{P}=0.02363 \mathrm{U}^{2}$ & $\mathrm{P}=0.02562 \mathrm{U}$ \\
& $0.34984 \mathrm{U}+0.47880$ & & $+1.23065 \mathrm{U}$ & +0.37465 & +0.36862 \\
& $\mathrm{Q}=1.84959 \mathrm{U}^{2}-$ & $\mathrm{Q}=0.53458 \mathrm{U}^{1.51947}$ & $\mathrm{Q}=0.33408 \mathrm{U}^{2}$ & $\mathrm{Q}=0.46801 \mathrm{U}^{2}$ & $\mathrm{Q}=0.58674 \mathrm{U}$ \\
$\mathrm{Q}$ & $1.83791 \mathrm{U}+0.61936$ & & $+0.20579 \mathrm{U}$ & +0.07253 & -0.09219 \\
& 0.0040 & 0.0720 & 0.0989 & 0.0534 & 0.0892 \\
\hline
\end{tabular}


From the table,we can see that improved GP modeling method establish the load model just like the traditional model,it also have higher fitting precision and less modeling time because it don't need to composition Modeling. The method will have more obvious advantages in the future.

\section{Conclusions}

This paper present an improved GP method for load modeling.Load model plays an important role in power system simulation. The Genetic Programming applied to the load modeling solves the problem of model identification based on artificial.According the examples, The improved GP have higher fitting accuracy and also shows the feasibility and effectiveness.Compared with the traditional method,the power system load model based on improved Genetic Programming provide a new approach to load modeling.

\section{References}

[1] Jian Zhang.Power System Load Model and Identification[M].Beijing:China Electric Power Press,2007.10-37.

[2] Yong Tang,Hongbin Zhang,Junxian Hou,Dongxia Zhang.A Synthesis Load Model with Distribution Network[J].Power System Technology,2007,05:34-38.

[3] John RKoza.Genetic programming:On the programming of the computers by the means of natural selection[M].Cambridge,MA:MIT Press, 1992.

[4] Jin Wang,Xinran Li.Power System Static Load Model Parameter Identification Based on Field Measurement[J].Hunan Electric Power, 2001,S1:11-13.

[5] Kangshun Li,Yuanxiang Li,Ruiming Tang,Aimin Zhou,Zhijian Wu.Application of Genetic Programming on Statistical Modeling[J].Journal of System Simulation, 2005,07:1597-1600.

[6] Aimin Zhou,Hongqing Cao,Lishan Kang,Yuzhen Huang.The Automatic Modeling of Complex Functions Based on Genetic Programming[J].Journal of System Simulation,2003,06;797-799.

[7] Niels Holger Gerhard Konstantin Provos.Statistical steganalysis[D].Computer Science and Engineering in the University of Michigan,2003.56-75.

[8] Xi Cui.The Application Study of Improved GP in Circuit Modeling[D].Ocean University of China, 2008. 